\title{
A INTEGRATED MODEL BASED ON AHP AND MAXIMIZING DEVIATION METHOD AND ITS APPLICATION
}

\author{
Zhanglin Peng \\ School of Management \\ Hefei University of Technology \\ Hefei, Anhui, China \\ E-mail: pengzhanglin@163.com \\ Shanlin Yang \\ School of Management \\ Hefei University of Technology \\ Hefei, Anhui, China \\ E-mail: hgdysl@gmail.com \\ Xiaojia Wang \\ School of Management \\ Hefei University of Technology \\ Hefei, Anhui, China \\ E-mail: tonysun800@sina.com
}

\section{ABSTRACT}

This study explores the potential of applying analytic hierarchy process (AHP) and maximizing deviation (MD) to determine the regional innovation capability of Chinese districts that need evaluation. Compared with some conventional single methods, the proposed combined model incorporates a much wider range of quantitative and qualitative criteria, and deals with a much more certain and uncertain information, and provides a more detailed and thorough research. Firstly, we use the Analytic hierarchy process to deal with uncertain information, then get the first weight vector, which is determined by the importance or priorities of the attributes or criterions; secondly, we use maximizing deviation method to handle some certain information, then we get the second weight vector, which is determined by the discrepancy of the attribute values; finally, we integrate these two weight vector and apply them in evaluating and ranking the regional innovation capability n 31 districts (provinces, municipalities \& Autonomous Regions) in China.

Keywords: analytic hierarchy process, maximizing deviation, regional innovation capability 


\section{Introduction}

As we known, Chinese economy's progress is very uneven, and there is a big gap among different districts, especially, between the eastern and western area. In some extent, the uneven economy development is resulted from the uneven science \& technology development. In Chinese Mainland, there are 31 provinces, municipalities \& Autonomous Regions (not include Hong Kong, Macao, and Taiwan). Due to different polices, more or less R\&D investment, different human resources, etc., the imbalance of the regional innovation capability has been produced among these districts. But how much the imbalance is? So we need to evaluate these regional innovation capabilities and compare them.

How should we evaluate them, maybe there are so much methods, for example Data envelopment analysis (DEA), the Evidential Reasoning (ER), Technique for Order Preference by Similarity to an Ideal Solution (TOPSIS), Fuzzy Theory, AHP, etc. when most of these methods deal with the MADM Problems, some problems such as that the evaluation results are close to each other, the evaluation values differ to each other scarcely, the difference between these evaluations is not obvious and so on can easily occur in practice. Although the multiple attribute decision model based on maximizing deviation can enlarge the evaluation values discrepancy for the optimizing and ranking of the decision making schemes, it only considers the variable weight caused by the discrepancy of the attribute values and neglects the weight of the evaluation indicator itself in practice, causing that the evaluation results easily deviate the actual results.

In this study, we combine both the Analytic Hierarchy Process (AHP: Saaty, 1977, 1980) and Maximizing Deviation Method (a mathematical theory) to address the regional innovation capability. Both are useful evaluation tools and are widely accepted methods for improving efficiency and productivity. The synergistic model provides an unequivocal and replicable tool and method in evaluating some units including both certain and uncertain information.

This study organizes the remaining structure as follows. In section 2, we will review some related literatures. Section 3 describes the specific objectives in this paper. Section 4 proposes the in integrated evaluation model based on AHP and MD, and gives detailed computational processes and steps. Section 5 gives a numerical experiment and result analysis.

\section{Literature Review}

\subsection{Analytic Hierarchy Process (AHP)}

Analytic Hierarchy Process (Saaty, 1977), (Saaty, 1980), (Saaty, 1986), (Saaty, 1990) is a multi-objective multi-criteria decision making method or approach based on pairwise comparisons for elements in a hierarchical decision model, and is presented to solve some decision making problems, such as choosing a best one in a set of competing alternatives, or ranking all potential alternatives. In this method or approach, the problem can be decomposed into a hierarchical structure including levels of factors or elements. Meanwhile, the strength of the influence of these factors or elements at a particular level can be measured by pairwise comparisons. It has strong axiomatic foundation which highlights: (1) the reciprocal property; (2) homogeneity; (3) dependence; and (4) expectations. The three typical principles of AHP are decomposition, comparative judgments, and synthesis of priorities. AHP is also a problem-solving framework, which 
can enable us to cope with the intuitive, the rational, the irrational, deal with certain and uncertain information, and combine objective and subjective judgments at the same time, when we make multi-criteria and multi-actor decisions or evaluations.

\subsection{Maximizing Deviation}

But in some extent, we often face a problem that the evaluation results are so close to each other, and the difference among all the results isn't significant obviously. In order to enlarge the evaluation results, (Yingming, 1997) presented a fully objective decision method-maximizing deviation method, and use them to evaluate about industrial economic benefits under multi-criteria, the evaluation results is very significant, exact, reliable without subjectivity. The method has been applied by many researchers, such as, (Wu \& Chen, 2007), (Wei, 2008) etc.

But, in practice, we often face a decision problem include certain information and uncertain information, so we not only need objective method to deal with certain information, and need other methods to handle uncertain information. Only by this way, we can get more exact, more precise, more reliable results. So, in this paper, we connect the AHP and maximizing deviation method, and use them to evaluate the Chinese regional innovation capability.

\section{Hypotheses/Objectives}

This study is mainly focused on the evaluation about the regional innovation capability in different Chinese districts. For simplicity, we just measure the innovation capability by science \& technology achievements, including Basic-Theory Research Achievements (BTRAs), Technical Achievements (TAs), and Soft S\&T Achievements (SSATs). The alternatives are 31 districts from Chinese Mainland, with 22 provinces, 4 municipalities, and 5 Autonomous Regions.

\section{Research Design/Methodology}

In this part, we construct a decision or evaluation model connected AHP with Maximizing Deviation Method. Firstly, we built the AHP model to get the priorities of criteria, that is weight vector of BTRAs, TAs and SSATs, then we add the weight vector to the original maximizing deviation decision model, in order to get the total weight vector including the attribute weight and variable weight. So the model built in this paper has two parts: (1) the AHP model; and (2) the integrated model based on AHP and Maximizing Deviation Model.

\subsection{The AHP Model}

Based on the AHP theory, and with the main kinds of S\&T Achievements and the current situation, three criteria are used to evaluate the regional innovation capability of different Chinese districts. These criteria are Basic Theory Achievements (BTAs), Technological Achievements (TAs) and Soft Science Achievements (SSAs).

The 31 districts in China Mainland assigned as the alternatives and their values are given, according to appendix 1, there are 3 years' statistic data which is from 2006 to 2008. 5 experts were invited to rank the 3 criteria according to Chinese S\&T development situation, then we can get the priorities of the criteria, that is, we also get the attribute 
weight vector. Next, we should add this weight vector to the original Maximizing Deviation Model, then work out the final weights of these 3 criteria.

\subsection{The integrated model based on AHP and Maximizing Deviation}

In conclusion, the main parts of the integrated model are as follows:

Step1: Determine all alternatives (Chinese cities or areas) and attributes (representing the regional innovation capability), and built a set of $A=\left\{A_{1}, A_{2}, \mathrm{~L}, A_{m}\right\}$ and a set of $G=\left\{G_{1}, G_{2}, \mathrm{~L}, G_{n}\right\}$.

Step2: Use AHP to work out the attribute weight or priority, and get the first weight vector $W=\left(w_{1}, w_{2}, \mathrm{~L}, w_{n}\right)^{T}$.

Step3: Construct the normalized and weighted decision matrix $C^{*}$, which be added $w_{j}$. Step4: Construct the maximizing deviation model, and use the Lagrange Function to get the second weight vector $W^{*}=\left(w^{*}{ }_{1}, w_{2}{ }_{2}, \mathrm{~L}, w_{n}{ }_{n}\right)^{T}$, then we get its normalization weight is $w_{j}^{*^{\prime}}$, that is, get the finally weight vector corresponding to each decision criteria.

Step5: Calculate the regional innovation capability of each city or area in China using the formula $E_{i}^{\prime}=w_{1}^{* \prime} w_{1} u_{i 1}+w_{2}^{*^{\prime}} w_{2} u_{i 2}+\mathrm{L}+w_{n}^{*^{\prime}} w_{n} u_{i n}$, then we can compare and rank them, and we can give some further analysis.

\section{Data/Model Analysis}

According to the AHP and the constructed criteria, we invited some related experts to make pairwise comparison, and get the priorities by Software SuperDecisions. Then we get the first weight vector $W=\left(w_{1}, w_{2}, \mathrm{~L}, w_{n}\right)^{T}$ is $W=(0.3125,0.6250,0.0625)^{T}$.

According to the first weight vector and the statistical data of S\&T Achievements from 31 districts (Provinces municipalities \&Autonomous Regions): 2006-2008, we use the integrated model to calculate the second weight vector, and use software Matlab by programming to solve the optimization model. Then we get e can get the 3 final weight vectors corresponding to 3 years respectively. They are $W^{*}=(0.3048,0.6228,0.0724)$, $W^{*}=(0.3062,0.6308,0.0630)$ and $W^{*}=(0.2525,0.6848,0.0627)$.

Next, we can get the ranking results.

\section{Limitations}

The paper has the following limitations: (1) due to some difficulties in collecting data of each district in recent years, so we have to adopt the data from 2006 to 2008. (2) in this paper, we give a rank about 31 alternatives, but we don't give more further quantitative analysis about why the model connection AHP and maximizing deviation is better than the single method, AHP or maximizing deviation method.

\section{Conclusions}

A combination evaluation model based on AHP and Maximizing Deviation Method has been presented and constructed in this paper, which not only can deal with certain information related to attribute values to get the variable weight of criteria, but also can handle uncertain information related to the importance of attributes themselves to get the International Symposium of 
attribute weight of criteria. In a word, the model can cope with kinds of information. Furthermore, the evaluation results will be significant.

Another important work in this paper is the application of this combination evaluation model. We apply it to evaluate the regional innovation capability in 31 Chinese districts, then compare them and analyze them. The evaluation and sorted results have shown that Chinese S\&T development had a so much obviously imbalance in 31 districts from 2006 to 2008 , especially, the western region had lower development in S\&T than eastern region, and lower than central region.

\section{Key References}

Saaty, T. L. (1977). A scaling method for priorities in hierarchical structures. Journal of Mathematical Psychology, 15(3), 234-281.

Saaty, T. L. (1980). The Analytic Hierarchy Process. New York, NY: McGraw-Hill.

Saaty, T. L. (1986). Axiomatic Foundation of the Analytic Hierarchy Process. Management Science, 32(7), 841-855.

Saaty, T. L. (1990). How to make a decision: The analytic hierarchy process. European Journal of Operational Research, 48(1), 9-26. doi:10.1016/03772217(90)90057-I

The Statistical Annual Report of Chinese S\&T Achievements of 2008. (2009). Retrieved from

http://www.tech110.net/uploadfile/statistics/uploadfile/200906/20090603025434 867.pdf

Wei, G.-W. (2008). Maximizing deviation method for multiple attribute decision making in intuitionistic fuzzy setting. Knowledge-Based Systems, 21(8), 833-836. doi:10.1016/j.knosys.2008.03.038

Wu, Z., \& Chen, Y. (2007). The maximizing deviation method for group multiple attribute decision making under linguistic environment. Fuzzy Sets and Systems, 158(14), 1608-1617. doi:10.1016/j.fss.2007.01.013

Yingming, W. (1997). Using the method of maximizing deviation to make decision for multiindices. Journal of Systems Engineering and Electronics, 8(3), 21-26. 